\title{
APPLICATION OF \\ MICROSTEREOLITHOGRAPHY \\ TECHNOLOGY IN \\ MICROMANUFACTURING
}

\section{Hongyi Yang, Gregory Tsiklos, Ronaldo Ronaldo, Svetan Ratchev}

School of Mechanical, Materials and Manufacturing Engineering,

University of Nottingham University Park Nottingham, NG7 2RD

\begin{abstract}
Stereolithography (SL) technology is the mostly widely used Rapid Prototyping (RP) process in which complex structures are fabricated in a layer-bylayer fashion. Currently Microstereolithography (MSL) is undergoing fast development. In this paper, the development of MSL and its application in micromanufacturing are reviewed. One promising direction for the development of MSL is the assembly-free process, through which a complex structure can be manufactured in a single process thus avoids the extra assembly procedure. With the assembly-free process, the manufacturing cost can be greatly reduced and the manufacturing reliability dramatically improved. The assembly-free process may play an important role in the future fabrication practice.
\end{abstract}

Key words microstereolithography, micromanufacturing, assembly free process

\section{Introduction}

The sterolithgraphy (SL) process is the first rapid prototyping technique which was patented in 1984. Currently it is widely used in various industrial and technological fields that require the direct manufacturing of three dimensional prototype parts [1, 2]. In SL process, small-size, high-resolution three-dimensional objects are built by superimposing a certain number of layers obtained by a light-induced and spaceresolved polymerization of a liquid resin into a solid polymer. The laser beam is focused and polymerisation occurs locally, which allows creating the shape of one layer of the object. When a layer is finished, fresh resin is spread on top of the already manufactured part of the object, and the light-induced solidification of the next layer is started. SL technique is regarded as a milestone in the manufacturing practice, because the layer-by-layer building process enables applying adding material process as compared to the removing of materials used by traditional techniques.

Microstereolithography (MSL) is the general designation of various microfabrication technologies with high-resolution based on the principle used in SL, which was proposed by lkuta et al. in 1993[3]. Ikuta et al. also named MSL as 
Micro-Photoforming, IH process, Spatial Forming, 3D Optical Modelling etc., depending on the different designs of the building apparatuses used in the manufacturing process. Compared with SL, MSL has a resolution of submicron scale for the $x-y-z$ translational stages, also the UV laser spot in MSL is finely focused to submicron scale, and thus MSL allows for polymerizations of layers with 1-10um thickness [4].

Modern micro-manufacturing industries are facing increasing requirements of miniaturization, customisation and integration in the fabrication of micro devices such as Micro-electro-mechanical systems, microfluidic systems. The MSL technology provides an ideal solution to such challenges due to its unique characters of high resolution, high liability and lower cost. Currently MSL is being exploited in different fields to cater for various new applications.

\section{State of the art}

The MSL system mainly consists of four parts: a laser source, a beam delivery system, a CAD design tool and computer-controlled precision $x-y-z$ stages, and a vat containing UV curable resin. After designing the 3D solid model with CAD software, the 3D model is sliced into a series of 2D layers with uniform thickness. The NC codes generated from each sliced $2 \mathrm{D}$ file are then executed to control the UV-beam scanning.

Based on the different beam delivery system used, two major beam scanning techniques have been developed: scanning MSL (or vector-by-vector MSL) and projection MSL (or integral MSL). Figure 1 schematically shows these two kinds of systems.

The Scanning MSL machines build the solid micro parts in a point-by-point and line-by-line fashion. A classical MSL apparatus is shown in Figure 1 (a), in which the laser beam is focused by a dynamic lens and deflected by two low-inertiagalvanometric on the surface of a resin system containing UV photoinitator, monomer, and other additives. An acousticoptical shutter cuts out the laser beam when a switch between the last polymerised segment and the next segment to be polymerised must be made without polymerization. To get a better resolution, the beam is focused more precisely in order to reduce the spot size to a few micrometers in diameter. Additional technological developments in the designed SL machines may be required. Constrained surface technique, Free surface technique are normally used in the commercial MSL systems. A main problem of the Conventional MSL is the limitations in terms of the minimum thickness of the resin layers during the layer preparation because of viscosity and surface tension. This problem was solved by two-photon MSL process recently, with this process the resin does not need to be layered. With two-photon MSL process, micro and even nano structure can be fabricated precisely [5].

Projection MSL builds 3D microstructure in a layer-by-layer fashion. Each layer is built by exposure through mask once, which significantly saves the time 
compared to the scanning MSL. A dynamic pattern generator is used to generate pattern projection for exposure curing (Figure $1(\mathrm{~b})$. When a beam passes through the projector, it contains the pattern of the layer and focused on the resin surface for the polymerization of the exposed areas. The time required to build a 3D structure only depends on the number of layers of the structure. Nowadays, two kinds of computer driven dynamic pattern generators, liquid crystal display (LCD) and Digital Light Processing (DLP) are used. For the LCD projector, an addressed LCD light valves array (made of liquid-crystal material) is used to control the on/off of the light, acting as a projector. The LCD technique has some intrinsic drawbacks, including large pixel sizes, low filling ratio, low switching speed ( $20 \mathrm{~ms})$, low optical density of the refractive elements during the OFF mode and the higher light absorption during the ON mode [6]. Such drawbacks limited further improvement of the LCD projection MSL system. In DLP projectors, the image is created by microscopically small mirrors laid out in a matrix on a semiconductor chip, known as a Digital Micromirror Device (DMD), which is a competing technology in the field of digital display. Each mirror represents one pixel in the projected image. The number of mirrors corresponds to the resolution of the projected image. High resolution DMD like $2800 \times 2100$ has been used in MSL. These mirrors can be repositioned rapidly, which is essential for switching between 'on' and 'off'. Using DMD, Sun etc. fabricated complex 3D microstructures with the smallest feature of $0.6 \mu \mathrm{m}$ [6]. Because of its capability in batch fabrication of 3D Microsystems, the dynamic projection MSL is a promising technology in micro manufacturing.

Currently efforts are also being made to incorporate a broad spectrum of materials into MSL fabrication for the manufacturing of micro-electro-mechanical systems with special functions. Metal, ceramic as well as polymer micro parts have been fabricated $[7,8]$. Similar to the SL of ceramic and metals, micro or even nanoceramic and metallic powders are mixed with photocurable resin for the MSL of ceramics and metals. Researches on functional photocurable polymers such as biodegradable polymers also have found applications in Tissue Engineering, drug delivery systems and so on $[9,10]$.

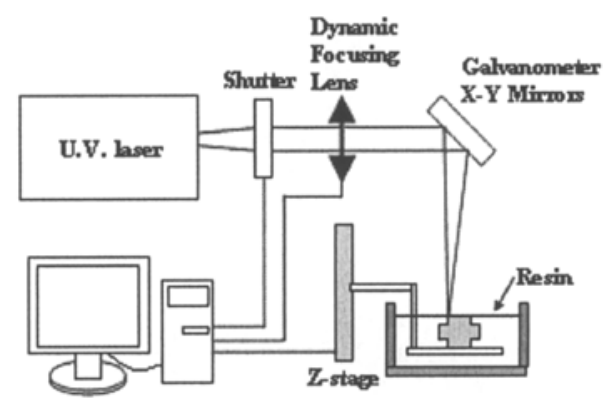

Fig. 1(a). Schematic view of MSL system: Scanning MSL 


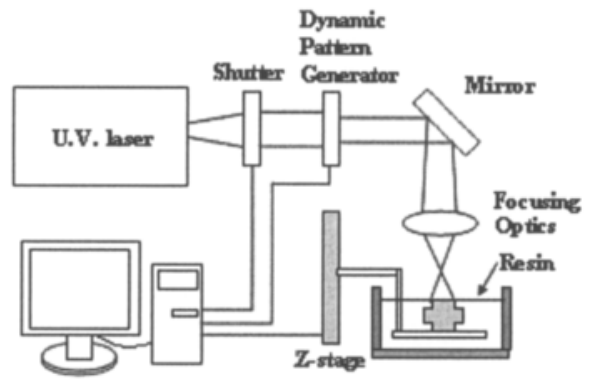

Fig. I(b). Schematic view of MSL system: Projection MSL.

\section{Case study}

In this study, the samples are fabricated by Perfactory MSL system of EnvisionTec, Germany. This projection MSL system has a DLP projector and has a high resolution of $7.5 \mu \mathrm{m}$ and visible light $(\lambda=\sim 475 \mathrm{~nm})$ was used as energy source. Figure 2 shows some micro struclures fabricaled with this system. From these pictures, we can see that with this technique complex structure with fine figures can be realized. Porous scaffolds (Fig $2 \mathrm{a}$, Fig $2 \mathrm{e}$ and $2 \mathrm{f}$ ) and designed micro structures (Fig $2 \mathrm{~b}$ and Fig $2 \mathrm{~d}$ ) can be used for tissue engineering scaffold, drug delivery system, micro fluidic and other micro scale applications.

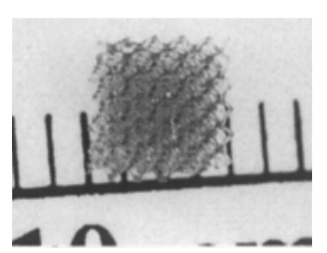

(a)

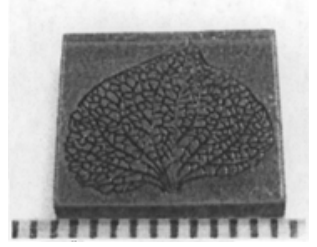

(d)

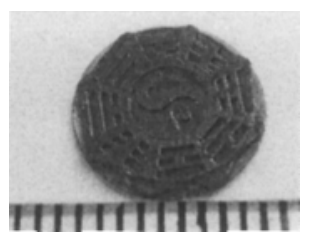

(b)

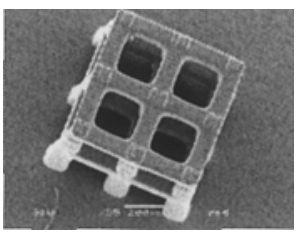

(e)

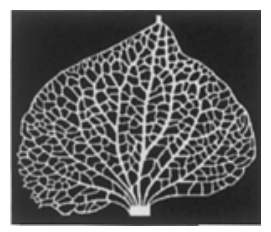

(c)

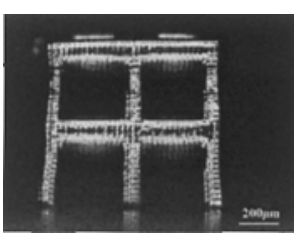

(f)

Fig. 2. Microstructures made by Perfactoryæ MSL 
Microsystems are usually complex, and composed of a number of unit devices to be fabricated separately and then assembled together. However, with the miniaturisation of the $\square$ icrosystems, the assembly process becomes more and more difficult, time-consuming, and expensive. It is estimated that assembling process takes $80 \%$ of the whole manufacturing costs in the Microsystems. Thus the assembly-free design represents $a$ new and promising direction in the manufacturing technology.

With MSL it is possible to fabricate complex free-forming three-dimensional microstructures in a single process, and the assembly process can be avoided. Typical example of assembly-free manufacturing has been published [11], such as the micro-fluidic systems which have seen wide applications in the area of microchemistry and microbiology. Generally, micro-fluidic systems have several components, such as the micro-channels, micro-pumps and micro-mixers. The traditional technologies used for micro-fluidic systems manufacturing are lithography, etching and micromachining [12]. Assembly process is needed to fabricate a closed system because of the micro fluidic structure can be only made on a 2D layer. Using MSL, 3D microstructure can be manufactured directly. Fig 3 shows a multi-analysis micro fluidic system with a size of $2 \times 10 \times 14 \mathrm{~mm}$ and $300 \mu \mathrm{m}$ fluidic channel inside. For each analysis part, there are two inlets, mixing channel, testing chamber and one outlet. The total manufacturing process for this structure was only taken less than 2 hours. The whole process was greatly simplified by reducing assembly process and other assistant processes compared with other traditional methods.

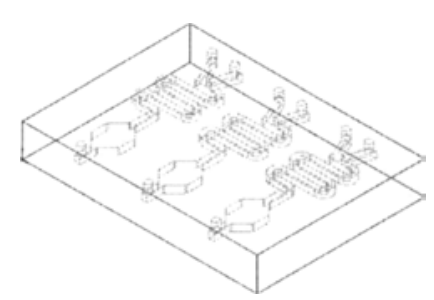

(a)

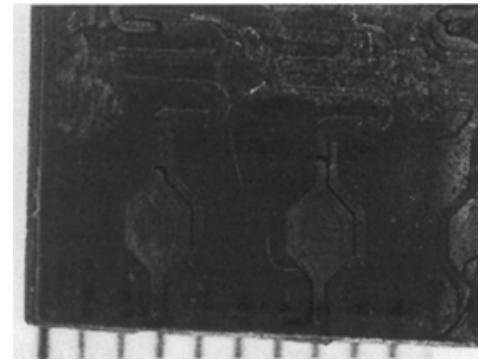

(b)

Fig. 3. Micro fluidic device made by Perfactory MSL: (a) designed structure and (b) Microscope photo.

\section{Conclusion and future works}

With MSL, complex micro structure can be realised with high resolution. Assembly-free process of micro systems can also be realized with the improvement of MSL, which is promising because of the reducing of microassembly cost. Especially, with the development of the projection MSL system with DLP projector, mass production can be realised. 
Current commercial SL or MSL techniques still have many technical limitations, such as low reliability and limited applicability of materials. Currently researches are working on several key issues to improve the MSL technology. The first is to improve the resolution of the fabrication process. The resolution is affected by many factors including machine, software, process, and resin related factors. The second is to search for new photo curable materials. Integration with other microfabrication technologies is also a promising direction for assembly-free process of multimaterials.

\section{Acknowledgements}

The authors are grateful to the Centre of Excellence in Customised Assembly (CECA) for supporting this work.

\section{References}

[1] C. Hull, Method for production of three-dimensional objects by stereolithography, US patent No. $4575330,1984$.

[2] J.C. Andre, M.A. Le, and O. de Wittee, French Patent, 8411241, 1984.

[3] K. Ikyta and K. Hirowatari, Real three dimensional microfabrication using stereo lithography and metal molding, Proc. IEEE MEMS, 1993, p $42-47$.

[4] V. K. Varadan, X. N. Jiang, V. V. Varadan, Microstereolithography and other fabrication techniques for 3D MEMS, Wiley, 2001, p105.

[5] S. Wu, J. Serbin, M. Gu, Two-photon polymerisation for three-dimensional microfabrication, Journal of Photochemistry and Photobiology A: Chemistry, 191, 1-11, (2006).

[6] C. Sun, N. Fang, D. Wu, X. Zhang, Projection micro-stereolithography using digta] micro-mirror dynamic mask. Sensors and Actuators A121,113-120, (2005).

[7] A. Bertsch, S. Jiguet and P. Renaud, Microfabrication of ceramic components by microstereolithography, J. Micromech. Microeng. 14, 197-203, (2004).

[8] K.F. O Connor, D.C. Nohns, W.A. Chattin, Method of combining metal and ceramic inserts into stereolithography components, United States Patent 5705117, 1998

[9] K.S. Anseth, A.T. Metters, S.J. Bryant, P.J. Martens, J.H. Elisseeff, C.N. Bowman, In situ forming degradable networks and their application in tissue engineering and drug delivery. Journal of Controlled Release, 78, p199-209, (2002).

[10] J.W. Lee, B. Kim, G. Lim and D.W. Cho, Scaffold Fabrication with Biodegradable Poly(propylene fumarate) Using Microstereolithography. Key Engineering Materials, 342$343, \mathrm{p} 141-144,(2007)$.

[11] H.W. Kang, I.H. Lee, D.W. Cho, Development of an assembly-free process based on virtual environment for fabricating $3 \mathrm{D}$ microfluidic systems using microstereolithography technology. Journal of Manufacturing Science and Engineering, 126, p766-771, (2004).

[12] P. Abgrall and A.M. Gue, Lab-on-chip technologies: making a microfluidic network and coupling it into a complete microsystem- a review, J. Micromech, Microeng, 17, R15-R49, (2007). 